\title{
Men's perceptions of PrEP use by their adolescent and young adult female partners in Tanzania in Tanzania: Findings from implementation science research
}

Population Council

Follow this and additional works at: https://knowledgecommons.popcouncil.org/departments_sbsr-hiv

Part of the Demography, Population, and Ecology Commons, Family, Life Course, and Society Commons, International Public Health Commons, and the Medicine and Health Commons How does access to this work benefit you? Let us know!

\section{Recommended Citation}

Population Council. 2017. "Men's perceptions of PrEP use by their adolescent and young adult female partners in Tanzania in Tanzania: Findings from implementation science research," DREAMS Tanzania Results Brief 5. Washington, DC: Population Council. 


\section{MEN'S PERCEPTIONS OF ORAL PRE-EXPOSURE PROPHYLAXIS USE BY THEIR ADOLESCENT AND YOUNG ADULT FEMALE PARTNERS IN TANZANIA FINDINGS FROM IMPLEMENTATION SCIENCE RESEARCH}

Oral pre-exposure prophylaxis (PrEP), which are antiretroviral medications taken by HIV-uninfected persons to prevent HIV, has been recommended by the World Health Organization for individuals at high risk for HIV. ${ }^{1}$ In Tanzania, PrEP has the potential to significantly reduce HIV acquisition among adolescent girls and young women (AGYW), one of the most vulnerable populations at risk for HIV. HIV prevalence in the country is 1.3 percent among adolescent girls 15-19 years old, tripling to 4.4 percent among young women $20-24$ years old. ${ }^{2-5}$

Prevailing gender norms about sexuality and relationship power dynamics have been shown to affect how women make decisions about the use of sexual and reproductive health products, including microbicides, condoms, and other contraceptives. ${ }^{6}$ Therefore, it is important to understand male partners' views of PrEP to inform how to effectively introduce PrEP among AGYW in Tanzania to ensure they use it correctly and consistently.

The Population Council, in collaboration with the National AIDS Control Programme (NACP) and CSK Research Solutions Ltd, conducted implementation science research in Tanzania to identify key considerations for introducing PrEP to AGYW. The study was implemented in two research sites-an urban setting in Dar Es Salaam and a rural setting in Mbeya district. This brief presents results on male partners' attitudes and perceptions of PrEP use by their AGYW partners.

\section{METHODS}

The study team conducted 16 in-depth interviews (IDIs) across the two research sites with men aged 18 or older who were married to or in a relationship with an adolescent girl (age 15-17) or young woman (age 18-24). IDIs were conducted in Kiswahili. Civil society organizations and service providers from health care facilities helped recruit male partners for the IDIs.

\begin{abstract}
their own and their partner's risk for HIV, their perceptions of PrEP use by AGYW and themselves, concerns they have about PrEP,

Trained research assistants used a standardized script to provide detailed information on PrEP to all participants prior to the interviews. Male partners were asked about their perceptions of
\end{abstract}

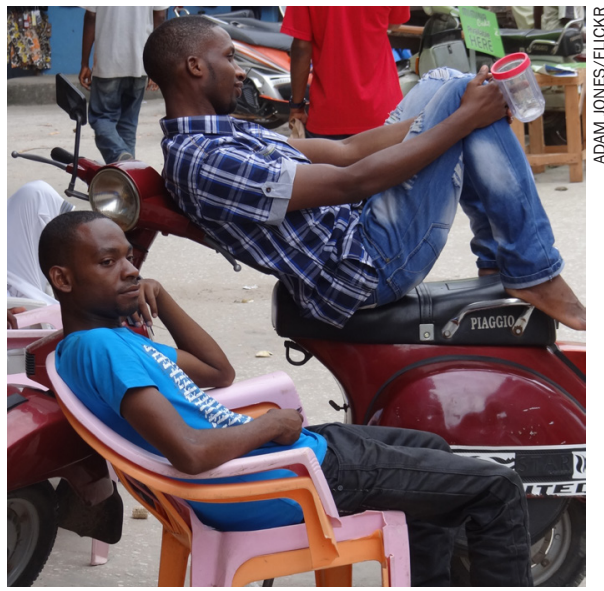

Men's support of PrEP use by AGYW is tied to their acknowledgement that both partners are at risk of HIV.

\section{KEY FINDINGS AND RECOMMENDATIONS}

Male partners recognize the need for PrEP due to relationship infidelity, which often accompanies low or inconsistent condom use.

Most male partners indicated that they would support their AGYW partner in using PrEP, but they must be engaged early in the decision-making process and provided with accurate information about PrEP.

Service providers can play an important role in reaching men and gaining their support for PrEP use.

Educating male partners about PrEP and engaging them in implementation activities should be part of PrEP roll-out strategies for AGYW. 
and how they would react to their AGYW partner taking PrEP. This study was approved by the Population Council Institutional Review Board (New York, USA) and the National Institute of Medical Research (Dar Es Salaam, Tanzania).

\section{RESULTS}

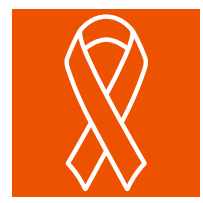

Men's support of PrEP use by AGYW is tied to their acknowledgement that both partners are at risk of HIV.

Male partners perceived that both partners engage in sexual intercourse outside of marriage or their relationship and that both partners could have occasional or regular partners besides their primary partner. Moreover, they acknowledged that they either did not use or used condoms inconsistently with their occasional or regular partners. They did not use condoms with primary partners because condom use is seen as a breach of trust or cheating. As such, male partners were aware that they, as well as their AGYW partner, were at risk for HIV.

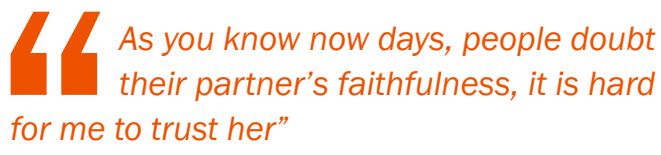

-Male partner, age 24, Mbeya

When one respondent was asked if he used condoms when having sex and why not, he said:

\section{Honestly speaking I don't. ... I trust \\ them and they both trust me...we don't use condoms" \\ -Male partner, age 24, Dar es Salaam}

The recognized risk of HIV due to relationship infidelity and inconsistent condom use resulted in most respondents expressing support for their female partner's use of PrEP.

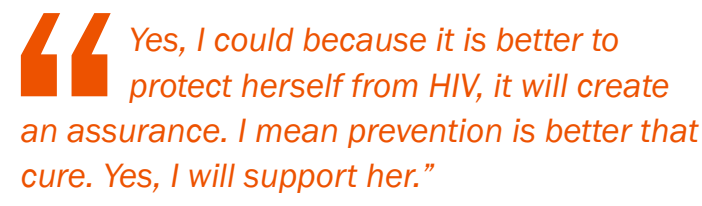

-Male partner, age 34, Dar Es Salaam

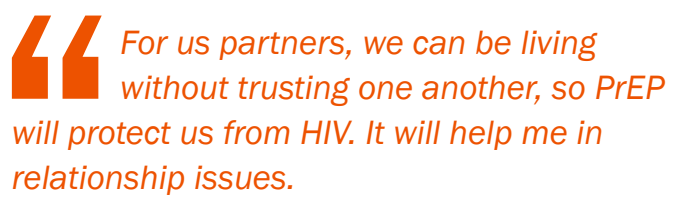

-Male partner, age 28, Mbeya

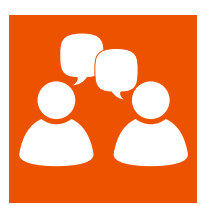

Early engagement in their partner's decision-making around PrEP is important for gaining male partners' support.

Male partners' support of their AGYW

partner's use of PrEP was often dependent on the condition that AGYW disclose their decision to use PrEP prior to actual use and engage their male partner in the decision-making process. Some noted that their approval

\section{WHO WERE THE STUDY PARTICIPANTS?}

Age ranged from 19 to 42 years

11 were married,

3 living with partner, and

2 never married

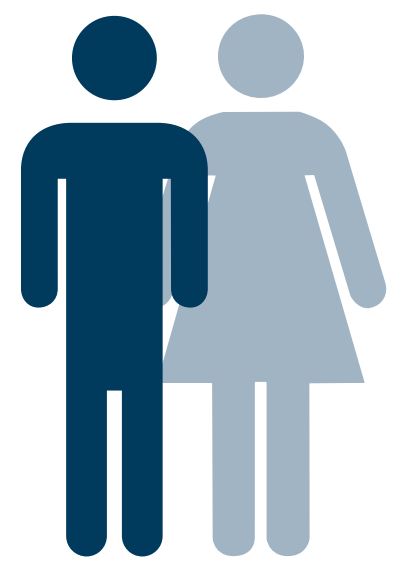

Over half completed secondary school, 5 primary school, and only 2 attained post secondary

Occupations: 5 were in business, 2 farming, 1 government, 1 student, and the rest varied 
was required in order for their AGYW partner to use PrEP. Male partners saw non-disclosure of PrEP use by their AGYW partner as extremely negative, as it would confirm their suspicion of infidelity and signify a lack of faith in the marriage or relationship. Moreover, lack of disclosure challenged their authority as head of the household.

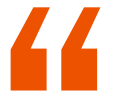

She must ask me about that [referring

to PrEP]... because if she doesn't tell and I come to realize that she is taking PrEP then I will lose trust in her."

-Male partner, age 26, Dar Es Salaam

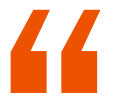

I will ask her many questions as to why she is taking PrEP without my knowledge; I will also investigate if she has another partner."

-Male partner, age 24, Mbeya

Instead, male respondents expressed an interest in being engaged in the decision-making process, which they felt was important to maintaining and strengthening relationship trust and stability. Being engaged early by their AGYW partner or being asked for their approval would shift male partners' thoughts about AGYW's reasons for taking PrEP from "she's being unfaithful" to "she's concerned about her health."

46 First is to get me involved. To involve me in what she wishes to do, which is to use these medications. So the most important thing is for me to be involved in the whole process"

-Male partner, age 42, Dar Es Salaam

When asked if he would like his partner taking PrEP and why, one respondent said:

4 Yes, I would like.... Simply because I have understood about it, and, it is the best way to protect ourselves from HIV and keep us safe."

-Male partner, age 28, Mbeya

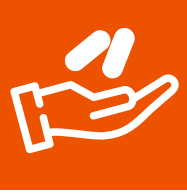

Male partners are interested in using PrEP to protect themselves.

When asked if they would be interested in taking PrEP, most men reported that they would want to take it for their own protection.

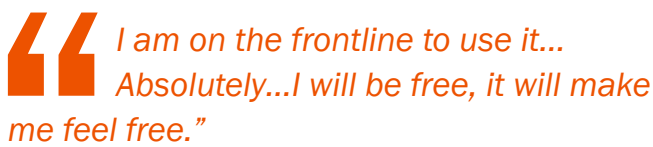

-Male partner, age 32, Dar Es Salaam

At the same time, however, respondents also acknowledged that their use of PrEP would induce doubt or suspicion about their fidelity and trust among their AGYW partner.

Interviewer:

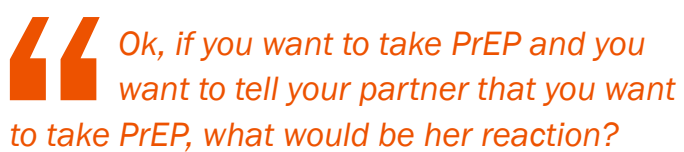

Respondent:

44 $\mathrm{Mmm}$ ! She is not going to trust me anymore; it will be so hard for her even if I educate her on PrEP."

-Male partner, age 30, Mbeya

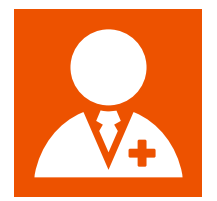

Couple counseling is seen as a facilitator for PrEP uptake and use. Male partners highlighted the importance of receiving accurate information and education about PrEP and its benefits directly from health service providers in order to facilitate their support of PrEP use by their AGYW partner.

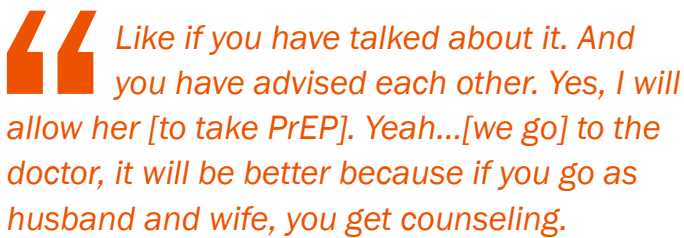

-Male partner, age 25, Dar Es Salaam 
Male partners also mentioned that service providers could play an important role in suggesting strategies on how they can actively support their partner's use of and adherence to PrEP. When asked what are things that he would do to support his partner's decision, one study participant responded:

\section{On my side, I would prefer to go to the doctor to get advice.}

-Male partner, age 26, Mbeya

\section{RECOMMENDATIONS}

- Engage male partners in PrEP implementation activities at an early stage so that they are aware of the benefits of PrEP; this will increase the likelihood that they will support their partner's decision to use PrEP.

- Involve male partners and couples in the development of education and sensitization materials and counseling messages about PrEP for individuals in relationships. Care should be taken that materials and messages do not exacerbate concerns about relationship trust and infidelity.

- Enhance the ability of health service providers to educate male partners of AGYW as well as couples about PrEP and to facilitate couple discussion and disclosure of PrEP use as needed. Counseling materials should be developed to aid service providers' provision of these services.

\section{REFERENCES}

${ }^{1}$ WHO. 2015. "Guideline on when to start antiretroviral therapy and on pre-exposure prophylaxis for HIV." Geneva, Switzerland: WHO.

${ }^{2}$ Pilgrim, N. et al. 2016. "Building evidence to guide PrEP introduction for adolescent girls and young women." Washington, DC: Population Council.

${ }^{3}$ Carroll, J. J. et al. 2016. "Gendered differences in the perceived risks and benefits of oral PrEP among HIVserodiscordant couples in Kenya," AIDS Care 2016: 1-7.

${ }^{4}$ Woodsong, C. et al. 2013. "Microbicide clinical trial adherence: insights for introduction," Journal of the International AIDS Society 16(1).

${ }^{5}$ Delany-Moretlwe, S. et al. 2016. "Planning for HIV preexposure prophylaxis introduction: lessons learned from contraception," Current Opinion in HIV and AIDS. 11(1): $87-93$.

${ }^{6}$ Tanzania Commission for AIDS (TACAIDS) et al. 2013. Tanzania HIV/ AIDS and Malaria Indicator Survey 201112. Dar es Salaam, Tanzania: TACAIDS, ZAC, NBS, OCGS, and ICF International.
For more information, contact Nanlesta Pilgrim (npilgrim@ popcouncil.org), Lou Apicella (lapicella@popcouncil.org), or Catherine Kahabuka (ckahabuka@cskresearch.com).
POPULATION COUNCIL

Ideas. Evidence. Impact.
The Population Council confronts critical health and development issues-from stopping the spread of HIV to improving reproductive health and ensuring that young people lead full and productive lives. Through biomedical, social science and public health research in about 50 countries, the Council works with our partners to deliver solutions that lead to more effective policies, programs, and technologies to improve lives worldwide. Established in 1952 and headquartered in New York, the Council is a nongovernmental, nonprofit organization with an international board of trustees. popcouncil.org

Suggested citation: Population Council. 2017. "Men's perceptions of PrEP use by their adolescent and young adult female partners in Tanzania in Tanzania: findings from implementation science research,” DREAMS Tanzania Results Brief 5. Washington, DC: Population Council. 